\title{
DIGITAL INPAINTING BASED ON THE MUMFORD-SHAH-EULER IMAGE MODEL
}

\author{
SELIM ESEDOGLU AND JIANHONG SHEN *
}

\begin{abstract}
Image inpainting is an image restoration problem, in which image models play a critical role, as demonstrated by Chan, Kang and Shen's recent inpainting schemes based on the bounded variation [10] and the elastica [9] image models. In the present paper, we propose two novel inpainting models based on the MumfordShah image model [37], and its high order correction — the Mumford-Shah-Euler image model. We also present their efficient numerical realization based on the $\Gamma$-convergence approximations of Ambrosio and Tortorelli $[1,2]$, and De Giorgi [18].
\end{abstract}

Key words. Inpainting, Bayesian, image model, Euler's elastica, $\Gamma$-convergence, elliptic equations, level-set method, connectivity principle.

AMS subject classifications. Primary: 94A08; Secondary: 68U10, 65K10.

1. Introduction. Inpainting is to fill in the missing or desired image information on domains where it is unavailable (see Figure 1.1). Such "blank" domains may be introduced by the scratches in a photograph, the aging of the canvas and oil of an ancient painting, and the occlusion by undesired objects in front of a scene of interest, etc.

Initially motivated by the manual retouching work of museum artists [16, 44], as first appeared in the pioneering work of Bertalmio, Sapiro, Caselles, and Ballester [6], digital inpainting has found its wide application in image processing, vision analysis, and the movie industry. Recent examples include: automatic scratch removal in digital photos and old films $[6,10]$, text erasing $[4,6,10,12]$, special effects such as object disappearance $[6,12]$, disocclusion [33], spatial/temporal zooming and super-resolution [4, 10, 43], lossy perceptual image coding [10], and removal of the laser dazzling effect [15], and so on. On the other hand, in the engineering literature, there also have been many earlier works closely related to inpainting, which include image interpolation [3, 25, 26], image replacement [21, 45] and error concealment $[22,27]$ in the communication technology. As scattered as the applications are, the methods for the inpainting related problems have also been very diversified, ranging from nonlinear filtering method, the Bayesian method, wavelets and spectral method, and the learning-and-growing method (especially for textures), etc.

The most recent approach to (non-texture) inpainting is based on the PDE method and Calculus of Variations, and can be classified into two categories. The first class is based on the simulation of micro-inpainting mechanisms. It includes the axiomatic approach of

* Selim Esedoglu is with: Institute of Mathematics and its Applications (IMA), University of Minnesota, Minneapolis, MN 55455, USA, esedoglu@ima.umn.edu. Jianhong (Jackie) Shen is with: School of Mathematics and IMA, University of Minnesota, Minneapolis, MN 55455, USA, jhshen@math.umn.edu, Tel. (612) 625-3570, Fax. (612) 626-2017. Send all comments to Shen. 


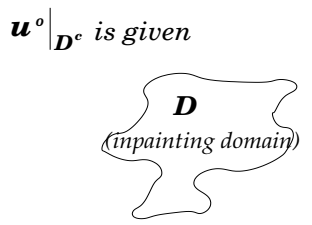

FIG. 1.1. For a typical inpainting problem, the image is missing on the inpainting domain $D$, and the available part $\left.u^{0}\right|_{D^{c}}$ is often noisy.

Caselles, Morel and Sbert [8], the transportation process [6] (the first high-order PDE model), the diffusion process [12], and their combination [5, 11]. The second category includes all variational models simulating the unique macro-inpainting mechanism: "best guess," or the Bayesian framework [17, 24, 36]. The latter includes the total variation model [13, 10, 40, 41], the functionalized elastica model [9,33], the value-and-direction joint model [4], and the active contour model based on Mumford and Shah's segmentation [43]. The Bayesian view on this category is also explained in the recent work of Chan, Kang, and Shen [9], and the underlying philosophy is indeed simple and intuitive: the way we (human inpainters) inpaint an incomplete picture mostly depends on two factors - how we read the existing part of the picture (i.e. data model), and what class of images we believe the picture belongs to (i.e. image model). For the latter, for examples, most windows and buildings have rectangular outlines, while tomatoes, potatoes, apples, and bottles in a kitchen are mostly round and smooth [38]. Such prior knowledge (or image model) is crucial for effectual inpainting.

The present work belongs to the latter category, and is intended to contribute to the further development of a key idea that first appeared in the recent works of Chan and Shen [10], and Tsai, Yezzi, and Willsky [43].

We propose two inpainting schemes based on the Mumford-Shah image model [37], and its high-order correction, the Mumford-Shah-Euler image model. The latter improves the former by replacing the embedded straight-line curve model with Euler's elastica [29]. Euler's elastica was first introduced by Mumford [35] into computer vision as a curve model, and has recently found its effective application in image inpainting through direct functionalization $[9,33]$.

The news about inpainting based on the Mumford-Shah image model (as first proposed in $[10,43])$ is mixed. The positive side is its lower order of complexity in terms of approximation and computation (Section 2). But unlike its effectiveness in the conventional application of segmentation and denoising (March and Dozio [30, 31], and Chan and Vese [14], for examples), the Mumford-Shah image model has its inherent deficiency for inpainting, as will be explained in Section 3 through two typical examples. The inpainting model based on the Mumford-Shah-Euler image model is designed to remedy such deficiency, and produce more 
natural visual effect (Section 4).

To turn these models to practical digital schemes, we seek help from the $\Gamma$-convergence results of Ambrosio and Tortorelli [1, 2] for the Mumford-Shah image model, and De Giorgi [18] for Euler's elastica. We explain why the $\Gamma$-convergence approximations appear to be more natural for inpainting than for segmentation and denoising. We also discuss the stable and efficient numerical implementation of the elliptic systems derived from these approximate models, as inspired by March and Dozio's similar earlier works on segmentation [30, 31]. These are written into Section 2 and 4.

Parallel to the level-set formulation in $[14,43]$ for Mumford-Shah segmentation, in Section 4, we present the level-set formulation for inpainting based on the Mumford-Shah-Euler image model, and discuss some of the related computational issues.

The summary and conclusion consist into Section 5. This completes our introduction to the main content and organization of the paper.

Throughout this paper, $\Omega$ denotes the entire image domain (in $R^{2}$ ), $x \in \Omega$ a general pixel, $D$ the inpainting domain where image is missing, and $u^{0}$ the available part of the image on $\Omega \backslash D$. For any multi-variable function or functional $F(X, Y)$, the symbol $F(X \mid Y)$ still means $F(X, Y)$, but with $Y$ fixed as known. This is to imitate the symbol of conditional probability or expectation in probability theory (but without normalization).

It is our best wish, that the current work, together with the efforts of all the authors whose works have been just mentioned, can generate further attention and interest from the applied mathematics community.

2. Inpainting via Mumford-Shah Image Model. A general variational inpainting model is to minimize an appropriate energy

$$
J\left[u \mid u^{0}, D\right] \quad \text { or } \quad J\left[u \mid u^{0} \text { given on } \Omega \backslash D\right] .
$$

By the Bayesian rationale [36], the energy consists of two parts:

$$
J\left[u \mid u^{0}, D\right]=\frac{\lambda}{2} \int_{D}\left(u-u^{0}\right)^{2}+E[u],
$$

where $E[u]$ encodes the image model, and the first term expresses the data model, of which we have assumed that

$$
u^{0}=u_{\text {original }}+\text { Gaussian white noise } n \text {. }
$$

Define $\lambda_{D}(x)=\lambda \cdot 1_{\Omega \backslash D}(x)$. Then the data model can be written as:

$$
\frac{1}{2} \int_{\Omega} \lambda_{D} \cdot\left(u-u^{0}\right)^{2} d x .
$$

The image model that Mumford and Shah [37] proposed for segmentation is the object-edge model:

$$
E[u, \Gamma]=\frac{\gamma}{2} \int_{\Omega \backslash \Gamma}|\nabla u|^{2} d x+\alpha H^{1}(\Gamma),
$$


where $\Gamma$ denotes the edge collection, $H^{1}$ the one dimensional Hausdorff measure, which generalizes the length notion for regular curves. In fact, in most segmentation applications, especially in numerical computation [14,43], $H^{1}(\Gamma)$ is conveniently substituted by length $(\Gamma)$, under the assumption that $\Gamma$ belongs to the Lipschitz class.

Therefore, by (2.1), inpainting based on the Mumford-Shah image model is to minimize

$$
J_{\mathrm{ms}}\left[u, \Gamma \mid u^{0}, D\right]=\frac{1}{2} \int_{\Omega} \lambda_{D}\left(u-u^{0}\right)^{2} d x+\frac{\gamma}{2} \int_{\Omega \backslash \Gamma}|\nabla u|^{2} d x+\alpha H^{1}(\Gamma) .
$$

The idea first appeared in the recent works of Tsai, Yezzi, Willsky [43], and Chan and Shen [10]. In [10], it was introduced as an alternative to the TV inpainting model. In [43], it serves as one of the major examples and applications for the numerical active contour algorithm.

In the current section, we propose to apply the $\Gamma$-convergence approximation of $E[u, \Gamma]$ to inpainting (2.3), as initially studied by Ambrosio and Tortorelli in the context of image segmentation. We shall explain why such approximation becomes more ideal for inpainting than for the original segmentation task. More importantly, the Ambrosio-Tortorelli approximation leads to a very simple inpainting algorithm, and its rapid numerical convergence.

In the Ambrosio-Tortorelli's $\Gamma$-convergence approximation, the edge set $\Gamma$ is approximated by its "signature" function $z_{\epsilon}$ :

$$
z_{\epsilon}: \Omega \rightarrow[0,1]
$$

which is nearly 1 almost everywhere except on a tiny tabular neighborhood (with width $O(\epsilon)$ ) of $\Gamma$, where it is close to 0 . Thus, up to a multiplicative normalization factor of order $O(1)$,

$$
\frac{1}{\epsilon}\left|1-z_{\epsilon}\right|^{p}, \quad p \geq 1
$$

is an approximation to the Dirac delta measure of $\Gamma-\delta_{\Gamma}(x)$ :

$$
\int_{\Omega} \delta_{\Gamma}(x) f(x) d x=\int_{\Gamma} f(x(s)) d s,
$$

where $s$ is the arc-length parameter. Therefore,

$$
\operatorname{length}(\Gamma)=\int_{\Omega} \delta_{\Gamma}(x) d x=\text { const. } \int_{\Omega} \frac{\left|1-z_{\epsilon}\right|^{p}}{\epsilon} d x .
$$

In fact, in Ambrosio-Tortorelli's approximation [1,2], $p$ is taken to be 2, and $z=z_{\epsilon}$ is designed to minimize

$$
E_{\epsilon}[z \mid u]=\frac{\gamma}{2} \int_{\Omega} z^{2}|\nabla u|^{2} d x+\alpha \int_{\Omega}\left(\epsilon|\nabla z|^{2}+\frac{(1-z)^{2}}{4 \epsilon}\right) d x
$$

(By [1, 2], $z^{2}$ should be $z^{2}+o(\epsilon)$.) Compared with the original image model (2.2), it is based 
on two (coupled) approximations (up to a multiplicative constant of order one)

$$
\begin{aligned}
\int_{\Omega \backslash \Gamma}|\nabla u|^{2} d x & \simeq \int_{\Omega} z^{2}|\nabla u|^{2} d x \\
\text { length }(\Gamma) & \simeq \int_{\Omega}\left(\epsilon|\nabla z|^{2}+\frac{(1-z)^{2}}{4 \epsilon}\right) d x .
\end{aligned}
$$

The disadvantage of the approximation is that the edges in the image are represented by diffuse interfaces as opposed to sharp ones. As mentioned above, the transition bandwidth of the edge signature $z$ is of order $\epsilon$. Even though $\epsilon$ needs to be small in theory, the numerical grid size imposes a lower bound - we found that for a reliable approximation and a stable scheme, the transition band must be sufficiently resolved. As a result, numerically feasible $\epsilon$ 's lead to quite diffuse transitions and increase the uncertainty of the edge locations.

But the enormous gain of the approximate model lies in that it is an elliptic integral of $z$ and $u$, and can be easily solved numerically using either the finite element or the finite difference methods (March [30], for example).

For segmentation, the disadvantage can sometimes be influential since high resolution of the edge layout is indeed needed (such as in the disocclusion application of Nitzberg, Mumford, and Shiota [38], and for accurately estimating the growth rate of a certain species of cells under a fixed microscopic view in medical image processing). For inpainting, however, the only concern is the restored image $u$, not the edge signature $z$. The low resolution of the edge set presented by $z$ has little influence on the restored image $u$. The sharpness of the real edges of $u$ is well preserved as long as $z$ almost vanishes along a narrow band covering the real edges. This makes the Ambrosia-Tortorelli approximation more ideal for inpainting. It leads to simple and fast computational schemes without losing the inpainting quality.

Another notable advantage over the level-set algorithm is that the $z$ function makes it unnecessary to assign multiphase level-set functions, or equivalently, it does not need to deal with the four-color problem mentioned in [14].

In summary, we propose to carry out inpainting by minimizing the $\Gamma$-convergence approximation of the exact model (2.3)

$J_{\epsilon}\left[u, z \mid u^{0}, D\right]=\frac{1}{2} \int_{\Omega} \lambda_{D}(x)\left(u-u^{0}\right)^{2} d x+\frac{\gamma}{2} \int_{\Omega} z^{2}|\nabla u|^{2} d x+\alpha \int_{\Omega}\left(\epsilon|\nabla z|^{2}+\frac{(1-z)^{2}}{4 \epsilon}\right) d x$.

Taking variations on $u$ and $z$ separately yields the Euler-Lagrange system:

$$
\begin{aligned}
\lambda_{D}(x)\left(u-u^{0}\right)-\gamma \nabla \cdot\left(z^{2} \nabla u\right) & =0 \\
\left(\gamma|\nabla u|^{2}\right) z+\alpha\left(-2 \epsilon \Delta z+\frac{z-1}{2 \epsilon}\right) & =0,
\end{aligned}
$$

with the natural adiabatic boundary conditions (due to the boundary integrals coming from integration-by-parts):

$$
\frac{\partial u}{\partial \vec{n}}=0, \quad \frac{\partial z}{\partial \vec{n}}=0
$$


Define two differential operators acting on $u$ and $z$ separately:

$$
\begin{aligned}
L_{z} & =-\nabla \cdot z^{2} \nabla+\lambda_{D} / \gamma \\
M_{u} & =\left(1+2(\epsilon \gamma / \alpha)|\nabla u|^{2}\right)-4 \epsilon^{2} \Delta .
\end{aligned}
$$

Given a pair of the current estimation $u$ and $z$, both $L_{z}$ and $M_{u}$ are positive definite elliptic operators. (Note: $L_{z}$ is indeed positive definite since in both theory and computation [1, 2], $z^{2}$ is replaced by $z^{2}+c$, where $c$ is a positive constant decaying faster than $\epsilon$ as $\epsilon \rightarrow 0$.) The Euler-Lagrange system (2.6) and (2.7) is simply written as

$$
L_{z} u=\left(\lambda_{D} / \gamma\right) u^{0} \quad \text { and } \quad M_{u} z=1
$$

This coupled system can be solved easily by any efficient elliptic solver and an iterative scheme such as the sequential strategy:

$$
L_{z^{(n-1)}} u^{(n)}=\left(\lambda_{D} / \gamma\right) u^{0} \quad \text { and } \quad M_{u^{(n)}} z^{(n)}=1,
$$

and the parallel strategy:

$$
L_{z^{(n-1)}} u^{(n)}=\left(\lambda_{D} / \gamma\right) u^{0} \quad \text { and } \quad M_{u^{(n-1)}} z^{(n)}=1
$$

Compared with March's approach for segmentation [30], this scheme is more stable and converges faster.

We have included in this section two numerical examples based on model (2.5) and its associated elliptic system (2.11). Figure 2.1 is a typical example in the inpainting literature [6, 10], of which the inpainting domain carries complicated topology (e.g., non-convex and not simply connected). The advantage of the numerical PDE approach, as compared with the dynamic programming algorithm of Masnou and Morel [33], is its capability of automatic filling regardless the shape and topology of the inpainting domain. The second example in Figure 2.2 shows an application of the inpainting technique for automatic text erasing.
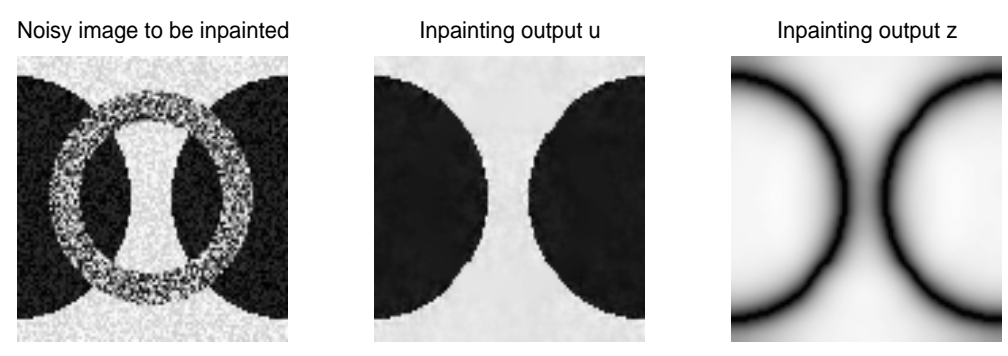

FIG. 2.1. Inpainting based on the $\Gamma$-convergence approximation (2.5) and its associated elliptic system (2.11). The annular inpainting domain is initially inpainted with a random guess for the iterative strategy (2.12) or (2.13). 

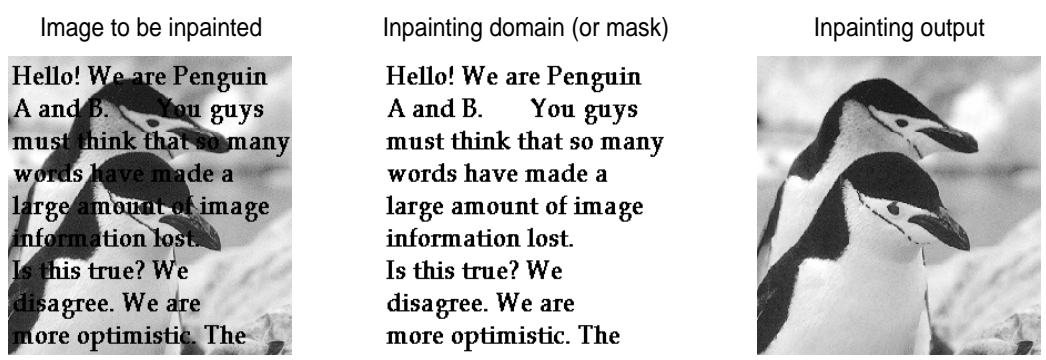

FIG. 2.2. Automatic text erasing by the inpainting model based on the $\Gamma$-convergence approximation (2.5) and (2.11).

3. Why is Mumford-Shah Insufficient for Inpainting. Numerous publications and numerical experiments $([14,30,43]$, just to name a few) have demonstrated the power of the Mumford-Shah image model for image segmentation and denoising. In this section, however, we discuss its insufficiency for inpainting. The shortcomings are first discovered by Chan and Shen [10], and are parallel to those of the TV inpainting model [10].

In Mumford and Shah's object-edge image model:

$$
E[u, \Gamma]=\frac{\gamma}{2} \int_{\Omega \backslash \Gamma}|\nabla u|^{2} d x+\alpha \text { length }(\Gamma)
$$

the preferable edge curves are those which have the shortest length. Therefore, without other types of constraints, it favors straight edges. In the classical segmentation and denoising application, the straight-line curve model is well balanced by the data model (or the fidelity term):

$$
\frac{\lambda}{2} \int_{\Omega}\left(u-u^{0}\right)^{2} d x
$$

For examples, we are still able to observe smoothly curved edges for blood cells in medical image processing, or for images with apples or tomatoes (check the top row of Figure 3.1).

The situation changes for inpainting, of which the image model is solely responsible for the reconstruction on the inpainting domain $D$. We explain the resulting defects through two typical phenomena and examples in the inpainting literature.

3.1. Emerging of artificial corners . The first artifact is the emerging of artificial corners, as clearly visible in Figure 3.1. Due to the lack of balance from the data model on the inpainting domain (the square domain in the bottom row, initially inpainted with a random guess), model (2.3) (or its approximation (2.5)) simply "draws" a straight line to complete the missing edge segment, since this costs the least energy according to the Mumford-Shah image model. As a result, the inpainted edge connects the existing edge segments in a non-tangent manner and leads to the two visible artificial corners. 


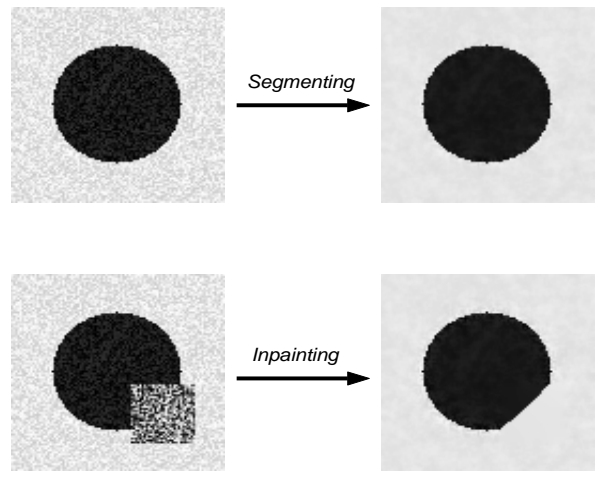

FIG. 3.1. The Mumford-Shah image model works well for segmentation (top row), but produces visible artificial corners when applied to inpainting (bottom row), due to the lack of balance from the data model (or the fidelity term) on the missing domain (Section 3.1).
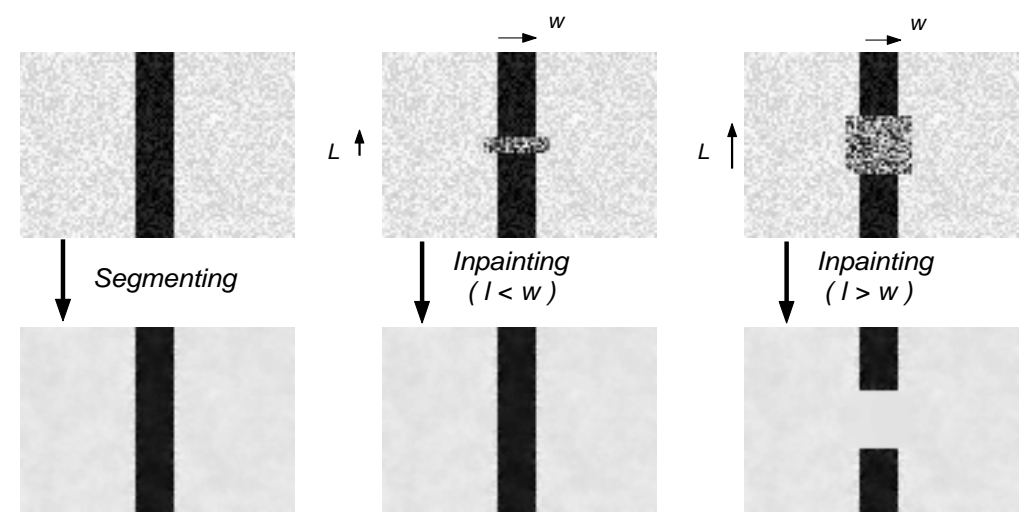

FIG. 3.2. The Mumford-Shah image model works well for segmentation (left), and for inpainting with small aspect ratio $l: w$ (middle), but fails to realize the Connectivity Principle [23,38] when the aspect ratio $l: w$ gets larger (right) (Section 3.2).

3.2. Violation of the Connectivity Principle. If the preceding shortcoming is more an issue of approximation order (like the linear approximation in numerical integration), then the second drawback is intrinsic to image and vision analysis.

Figure 3.2 shows that as the aspect ratio increases between the size $(l)$ of the inpainting domain and that $(w)$ of the object, the Mumford-Shah image model prefers the disconnection of separated parts. This is because, as $l$ exceeds $w$, long distance connection gets more expensive. For instance, let us sketchily analyze the third column $(l>w)$ : inside the inpainting domain, according to energy (2.3), a connected output would cost $2 \alpha l$, while the disconnection only costs $2 \alpha w$ (since $u$ is piecewise constant in this example).

In Chan, Kang and Shen's work $[9,12]$, this phenomenon is said to violate the Connec- 
tivity Principle. In image and vision analysis, this principle should be generally enforced on the consideration of two factors.

(i) The vision psychology (Kanisza [23], Nitzberg, Mumford, and Shiota [38]) shows that human observers mostly seem to prefer the connected outputs, and are not so sensitive to the aspect ratio.

(ii) In applications, an image often contains "objects" with a large dynamic range of scales. Thus in most inpainting problems $[5,10]$, it is commonly observed that "slim" objects such as decorating lines, thin stripes, or simple fiber-like textures are broken by an inpainting domain even though the latter has a moderate size to human observers. Thus connection should be generally encouraged.

Though the two artifacts (Figure 3.1 and 3.2) wear different masks in terms of their visual effects, they are rooted in the same soil - the insufficiency of the straight-line curve model embedded within the Mumford-Shah image model (2.2). Our next image model thus incorporates an improved curve model - Euler's elastica [9, 35], and is called the MumfordShah-Euler image model for the ease of reference.

Finally, we must point out that, despite the artifacts that the Mumford-Shah image model may produce, it is still an attractive one for digital inpainting due to the lower complexity of the associated differential equations and their fast computational schemes. It can at least supply high-quality initial guesses for computationally more costly inpainting schemes. On the other hand, for the recent noval applications in digital zooming and super-resolution [10], such lower order model is indeed already sufficient due to the local nature of these problems.

4. Inpainting via Mumford-Shah-Euler Image Model. As concluded from the discussion, the key to remedying the insufficiency of the Mumford-Shah image model is to improve its embedded curve model. As initially introduced into computer vision by Mumford [35], Euler's elastica curve model

$$
e(\Gamma)=\int_{\Gamma}\left(\alpha+\beta \kappa^{2}\right) d s=\alpha \text { length }(\Gamma)+\beta \int_{\Gamma} \kappa^{2} d s
$$

is a high order correction to the straight-line one. Here $\kappa$ denotes the curvature, $d s$ the length element, and $\alpha$ and $\beta$ two tunable positive weights. The energy was first studied by Euler to model the shape of a thin and torsion-free rod [29]. In approximation theory, Birkhoff and De Boor [7] referred to it as "nonlinear spline" for data fitting or interpolation, as compared to the classical polynomial based (linear) splines. Recently, Masnou and Morel [33], and Chan, Kang, and Shen [9] "lifted" the elastica curve model to an image model by direct functionalization, and applied it to disocclusion and image inpainting.

We shall call the modified energy

$$
E[u, \Gamma]=\frac{\gamma}{2} \int_{\Omega \backslash \Gamma}|\nabla u|^{2} d x+e(\Gamma)=\frac{\gamma}{2} \int_{\Omega \backslash \Gamma}|\nabla u|^{2} d x+\int_{\Gamma}\left(\alpha+\beta \kappa^{2}\right) d s
$$


the Mumford-Shah-Euler image model in this paper. The corresponding inpainting model becomes:

$$
J_{\mathrm{mse}}\left[u, \Gamma \mid u^{0}, D\right]=\frac{1}{2} \int_{\Omega} \lambda_{D}\left(u-u^{0}\right)^{2} d x+\frac{\gamma}{2} \int_{\Omega \backslash \Gamma}|\nabla u|^{2} d x+\int_{\gamma}\left(\alpha+\beta \kappa^{2}\right) d s .
$$

For a given edge layout $\Gamma$, the Euler-Lagrange equation for $J_{\mathrm{mse}}\left[u \mid \Gamma, u^{0}, D\right]$ is

$$
\lambda_{D}(x)\left(u-u^{0}\right)+\gamma \Delta u=0, \quad x \in \Omega \backslash \Gamma,
$$

with the adiabatic condition along $\Gamma: \partial u / \partial \vec{n}=0$. For the solution $u$, the Euler-Lagrange equation for $J_{\mathrm{mse}}\left[\Gamma \mid u, u^{0}, D\right]$ can be worked out to be $[9,35,28]$ :

$$
\left[\frac{\gamma}{2}|\nabla u|^{2}+\frac{\lambda_{D}}{2}\left(u-u^{0}\right)^{2}\right]_{\Gamma}+\alpha \kappa-\beta\left(2 \frac{d^{2} \kappa}{d s^{2}}+\kappa^{3}\right)=0
$$

where, given an arbitrary orientation (i.e. tangent direction $\vec{t}$ ) of $\Gamma, \kappa=|d \vec{t} / d s|$ (and thus $d \vec{t} / d s=\kappa \vec{n})$ is the curvature, and $[\phi]_{\Gamma}$ denotes the jump of a scalar function $\phi$ across the normal direction $\vec{n}$ : for any $x \in \Gamma$,

$$
[\phi]_{\Gamma}(x)=\lim _{\epsilon \rightarrow 0^{+}}(\phi(x+\epsilon \vec{n})-\phi(x-\epsilon \vec{n})) .
$$

For simplicity, we have assumed that the edge layout $\Gamma$ is smooth enough.

Computationally, the elliptic equation (4.4) for updating $u$ can be easily solved. The curve equation (4.5), on the other hand, leads to the active contour evolution:

$$
\frac{d x}{d t}=\left(\alpha \kappa-\beta\left(2 \frac{d^{2} \kappa}{d s^{2}}+\kappa^{3}\right)+\left[\frac{\gamma}{2}|\nabla u|^{2}+\frac{\lambda_{D}}{2}\left(u-u^{0}\right)^{2}\right]_{\Gamma}\right) \vec{n} .
$$

Due to the presence of the curvature term and its second derivative, the numerical implementation of (4.6) is much more involved than the conventional mean curvature motion. In what follows, we discuss two approaches: the level-set method of Osher and Sethian [39], and the method similar to Section 2 based on the $\Gamma$-convergence approximation of De Giorgi [18].

4.1. The level-set formulation of Osher and Sethian. Here we present the level-set method for a two-phase situation. The recent work of Chan and Vese [14] contains a detailed explanation of the multi-phase level-set method for the Mumford-Shah segmentation model.

In the level-set formulation of Osher and Sethian [39], the edge layout $\Gamma$ is embedded as the zero level-set of a Lipschitz continuous function $\phi(x): \Gamma=\{x: \phi(x)=0\}$. This redundant representation is then trimmed by the Heaviside functions:

$$
H_{+}(\phi)=\left\{\begin{array}{ll}
1 & \phi>0 \\
0 & \phi \leq 0
\end{array} \quad \text { and } \quad H_{-}(\phi)=\left\{\begin{array}{ll}
1 & \phi<0 \\
0 & \phi \geq 0
\end{array} .\right.\right.
$$

Both numerically and theoretically, they are understood as the limit (as $\epsilon$ tends to $0^{+}$) of:

$$
H_{\epsilon}(\phi)=\left\{\begin{array}{ll}
1 & \phi>\epsilon \\
0 & \phi \leq \epsilon
\end{array} \quad \text { and } \quad H_{-\epsilon}(\phi)=\left\{\begin{array}{ll}
1 & \phi<-\epsilon \\
0 & \phi \geq-\epsilon
\end{array} .\right.\right.
$$


Then the Mumford-Shah-Euler image model (4.2) has a natural representation under the levelset function $\phi$. First,

$$
\int_{\Omega \backslash \Gamma}|\nabla u|^{2} d x=\int_{\Omega} H_{+}(\phi)|\nabla u|^{2} d x+\int_{\Omega} H_{-}(\phi)|\nabla u|^{2} d x .
$$

For the elastica curve model, according to the similar idea of the co-area formula in the space of bounded variations (Giusti [19]), we have (as applied to the BV function $H_{+}(\phi)$ ):

$$
\begin{aligned}
\int_{\Gamma}\left(\alpha+\beta \kappa^{2}\right) d s & =\int_{\Omega}\left(\alpha+\beta\left(\nabla \cdot\left[\frac{\nabla H_{+}(\phi)}{\left|\nabla H_{+}(\phi)\right|}\right]^{2}\right)\left|\nabla H_{+}(\phi)\right| d x\right. \\
& =\int_{\Omega}\left(\alpha+\beta\left(\nabla \cdot\left[\frac{\nabla \phi}{|\nabla \phi|}\right]^{2}\right)|\nabla \phi| \delta(\phi) d x .\right.
\end{aligned}
$$

Here $\delta$ is the Dirac delta function, and the derivatives are understood formally, or in the weak-limit sense via the Gaussian convolution (denoted by $*$ ) kernel $G_{\sigma}(x)$ :

$$
\frac{\partial}{\partial x_{i}}=\lim _{\sigma \rightarrow 0^{+}} \frac{\partial}{\partial x_{i}} \circ G_{\sigma} *
$$

for $i=1,2$. In numerical implementation, as Chan and Vese [14] practiced, the Dirac delta function is replaced by its $\epsilon$ - approximation $\delta_{\epsilon}$, for example,

$$
\delta_{\epsilon}(x)=\frac{1}{\epsilon} g\left(\frac{x}{\epsilon}\right)
$$

for any numerically friendly positive function $g$ with a bell-shape and normalized total integral.

Therefore, the level-set representation of $J_{\mathrm{mse}}\left[u, \Gamma \mid u^{0}, D\right]$ becomes $J_{\mathrm{mse}}\left[u, \phi \mid u^{0}, D\right]$ as assembled from (4.7), (4.8), and the original fidelity term, which is now expressed by

$$
\frac{1}{2} \int_{\Omega} \lambda_{D}\left(u-u^{0}\right)^{2} d x=\frac{1}{2} \int_{\Omega} H_{+}(\phi) \lambda_{D}\left(u-u^{0}\right)^{2} d x+\frac{1}{2} \int_{\Omega} H_{-}(\phi) \lambda_{D}\left(u-u_{0}\right)^{2} d x .
$$

For a fixed level-set function $\phi$, variation of $J_{\mathrm{mse}}\left[u \mid \phi, u^{0}, D\right]$ on $u$ gives

$$
\lambda_{D}\left(u_{ \pm}-u^{0}\right)+\gamma \Delta u_{ \pm}=0, \quad \text { on } \quad \pm \phi>0,
$$

separately for $u_{ \pm}$with the adiabatic boundary condition: $\partial u_{ \pm} / \partial \vec{n}=0$. Then the combination of the formulae in Chan, Kang, and Shen [9] and Chan and Vese [14] expresses the gradient descent equation of $J\left[\phi \mid u_{ \pm}, u^{0}, D\right]$ as

$$
\begin{aligned}
& \frac{\partial \phi}{\partial t}=\delta_{\epsilon}(\phi)\left[\nabla \cdot \vec{V}-\left(\frac{\gamma}{2}\left(\left|\nabla u_{+}\right|^{2}-\left|\nabla u_{-}\right|^{2}\right)+\frac{\lambda_{D}}{2}\left(\left(u_{+}-u^{0}\right)^{2}-\left(u_{-}-u^{0}\right)^{2}\right)\right)\right] \\
& \vec{V}=\left(\alpha+\beta \kappa^{2}\right) \vec{n}-\frac{2 \beta}{|\nabla \phi|} \frac{\partial(\kappa|\nabla \phi|)}{\partial \vec{t}} \vec{t}
\end{aligned}
$$


Here

$$
\vec{n}=\frac{\nabla \phi}{|\nabla \phi|}, \quad \vec{t}=\vec{n}^{\perp}, \quad \frac{\partial}{\partial \vec{t}}=\vec{t} \cdot \nabla, \quad \text { and } \quad \kappa=\nabla \cdot\left[\frac{\nabla \phi}{|\nabla \phi|}\right]
$$

(Note: due to the parity of $\vec{t}$ in the formula, it makes no difference whether one takes $\vec{t}$ or $-\vec{t}$.)

Since the numerical Dirac delta function $\delta_{\epsilon}$ has a non-zero bandwidth, to evolve $\phi$ by (4.10), both $u_{+}$and $u_{-}$have to be continuously extended across the edge set: $\Gamma: \phi=0$ (to at least cover the support of $\delta_{\epsilon}(\phi(x))$. Various computational techniques are available to carry out the extension, as discussed in detail in [14]. For the flux flow $\vec{V}$, one can benefit from the numerical (finite differencing) techniques described in [9].

We refer to Chan and Vese's recent works [14] for further detail regarding other computational issues such as the re-initialization process. Due to the less common fourth-order (nonlinear) term related to the curvature, the level-set computation is however costly and slow.

Thus, in the coming subsection, we seek an alternative computational strategy that is based on the "elliptic" approximation of the curvature term, as initially proposed by De Giorgi [18].

4.2. The $\Gamma$-convergence approximation of De Giorgi. De Giorgi [18] proposed to approximate Euler's elastica curve model

$$
e(\Gamma)=\int_{\Gamma}\left(\alpha+\beta \kappa^{2}\right) d s,
$$

by an elliptic integral of the signature $z$ (the two constants $\alpha$ and $\beta$ may vary):

$$
E_{\epsilon}[z]=\alpha \int_{\Omega}\left(\epsilon|\nabla z|^{2}+\frac{W(z)}{4 \epsilon}\right) d x+\frac{\beta}{\epsilon} \int_{\Omega}\left(2 \epsilon \Delta z-\frac{W^{\prime}(z)}{4 \epsilon}\right)^{2} d x
$$

where $W(z)$ can be the symmetric double-well function

$$
W(z)=\left(1-z^{2}\right)^{2}=(z+1)^{2}(z-1)^{2} .
$$

Unlike the choice of $W(z)=(1-z)^{2}$ in the previous section of the Mumford-Shah image model, here, more as in the level-set method, the edge layout $\Gamma$ is embedded as the zero levelset of $z$. Asymptotically, as $\epsilon \rightarrow 0^{+}$, a boundary layer grows to realize the sharp transition between the two well states $z=1$ and $z=-1$.

As March and Dozio did for segmentation [31], based on the De Giorgi approximation (4.12), we are able to replace the inpainting model (4.3) by an elliptic energy on $u$ and $z$ :

$$
J_{\epsilon}\left[u, z \mid u^{0}, D\right]=\frac{1}{2} \int_{\Omega} \lambda_{D}\left(u-u^{0}\right)^{2} d x+\frac{\gamma}{2} \int_{\Omega} z^{2}|\nabla u|^{2} d x+E_{\epsilon}[z]
$$


with $E_{\epsilon}$ specified in (4.12). The remarkable difference between segmentation and inpainting can be traced to the discussion in Section 3. For segmentation, as March and Dozio practiced, the ratio $\beta: \alpha$ can be as small as $O\left(h^{2}\right)$, with $h$ denoting the distance between two nearest neighboring pixels. This is because, as clearly demonstrated in the top row of Figure 3.1, even without the curvature term (i.e. the conventional Mumford-Shah model with $\beta=0$ ), the optimal edge layout can be as smooth as one expects, due to the balance of the fidelity term. For inpainting, however, with the defects described in Section 3 in mind, we have to increase $\beta$ to substantially enhance the role of the curvature term on the inpainting domain. In fact, our numerical experiments suggest that $\beta: \alpha$ should be of order $O(1)$. Such large $\beta$ dramatically increases numerical instability and thus restricts the stable size of marching steps. It is a major computational barrier that one has to overcome for realistic digital inpainting applications.

Let us work out the Euler-Lagrange system. For a given edge signature $z$, variation on $u$ in $J_{\epsilon}\left[u \mid z, u^{0}, D\right]$ gives

$$
\lambda_{D}\left(u-u^{0}\right)-\gamma \nabla \cdot\left(z^{2} \nabla u\right)=0,
$$

with the adiabatic boundary condition $\partial u / \partial \vec{n}=0$ along $\partial \Omega$. Then the steepest decent marching of $z$ for $J_{\epsilon}\left[z \mid u, u^{0}, D\right]$ is given by (also see [31])

$$
\begin{aligned}
\frac{\partial z}{\partial t} & =-\gamma|\nabla u|^{2} z+\alpha g+\frac{\beta w^{\prime \prime}(z)}{2 \epsilon^{2}} g-4 \beta \Delta g, \\
g & =2 \epsilon \Delta z-\frac{W^{\prime}(z)}{4 \epsilon},
\end{aligned}
$$

again with the Neumann adiabatic conditions along the boundary $\partial \Omega$ :

$$
\frac{\partial z}{\partial \vec{n}}=0, \quad \text { and } \quad \frac{\partial g}{\partial \vec{n}}=0 .
$$

Eq. (4.16) is of fourth-order for $z$, with the leading head

$$
-8 \epsilon \beta \Delta^{2} z \text {. }
$$

Thus, to ensure stability, an explicit marching scheme would require

$$
\Delta t=O\left(\frac{(\Delta x)^{4}}{\epsilon \beta}\right) .
$$

There are a couple of ways to stably increase the marching size. First, as inspired by Marquina and Osher [32], one could add an automatic "time corrector" $T$ :

$$
\frac{\partial z}{\partial t}=T(\nabla z, g \mid u)\left(-\gamma|\nabla u|^{2} z+\alpha g+\frac{\beta w^{\prime \prime}(z)}{2 \epsilon^{2}} g-4 \beta \Delta g\right),
$$

where $T(\nabla z, g, \mid u)$ is a suitable positive scalar, for example, $T=|\nabla z|$, as inspired by the mean-curvature motion literature. The second alternative is to turn to implicit or semi-implicit schemes. Eq. (4.16) can be rearranged to

$$
\frac{\partial z}{\partial t}+\gamma|\nabla u|^{2} z-2 \alpha \epsilon \Delta z+8 \beta \epsilon \Delta^{2} z=-\frac{\alpha}{4 \epsilon} W^{\prime}(z)+\frac{\beta W^{\prime \prime}(z)}{2 \epsilon^{2}} g+\frac{\beta}{\epsilon} \Delta W^{\prime}(z),
$$


Or simply

$$
\frac{\partial z}{\partial t}+L_{u} z=f(z)
$$

where $L_{u}$ denotes the positive definite elliptic operator ( $u$-dependent)

$$
L_{u}=\gamma|\nabla u|^{2}-2 \alpha \epsilon \Delta+8 \beta \epsilon \Delta^{2}
$$

and $f(z)$ the entire right hand side of (4.18). Then a semi-implicit scheme can be designed as: at each discrete time step $n$,

$$
\left(1+\Delta t L_{u}\right) z^{(n+1)}=z^{(n)}+\Delta t f\left(z^{(n)}\right)
$$

where the positive definite operator $1+\Delta t L_{u}$ is numerically inverted based on many fast solvers [20, 42].

Remarkably, the computational challenge does not end in the $4^{\text {th }}$ order Eq. (4.10) or (4.16). There is an extra layer of inherent difficulty with the inpainting model (4.3) the energy has many local minima! As in the notorious example of Molecular Dynamics (MD) where the energy has too many local minima, the steepest-decent based marching strategy (4.10) or (4.16), even if equipped with robust, stable, and efficient numerical schemes, can be easily trapped and stagnate at the local energy "wells." Such stagnation can fail to carry out visually meaningful inpainting as discussed in Section 3, for instance, the failure to realize the Connectivity Principle.

This phenomenon does not bother much in segmentation and denoising. The reason is quite the same as given in Section 3. For segmentation and denoising, the data model, or equivalently, the fidelity term in the energy, confines all admissible inpaintings $u$ within a small "ball" in $L^{2}(\Omega)$ centered at $u^{0}$, inside whose limited space, there are only one or very few energy wells. But for inpainting, we only know a "projection" (in $L^{2}(\Omega)$ ) of the entire image, and all admissible inpaintings $u$, despite the fidelity term, occupy an infinite "cylinder" (in $L^{2}(\Omega)$ ), inside which the energy can have many energy wells. To visualize this discussion, we have treated $L^{2}(\Omega)$ as $R^{3}$, and impressionistically plotted the situation in Figure 4.1.

Therefore, in the subsequent future sibling work, we shall discuss in much detail and more systematically how to computationally overcome such energy barriers. The potential techniques include: Gaussian-smoothing (as in Molecular Dynamics [34]), the multiresolution approach (as in Tsai, Yezzi, and Willsky [43]), the re-initialization or sharpening technique (as in the level-set method of Osher and Sethian [39]), and the CDD technique (curvature driven diffusion, as in Chan and Shen [12]).

We end this section by showing in Figure 4.2 and 4.3 two examples based on the MumfordShah-Euler image models.

5. Summary and Conclusion. In this paper, following the philosophy of the Bayesian and variational image restoration theory, we have proposed two digital inpainting models 


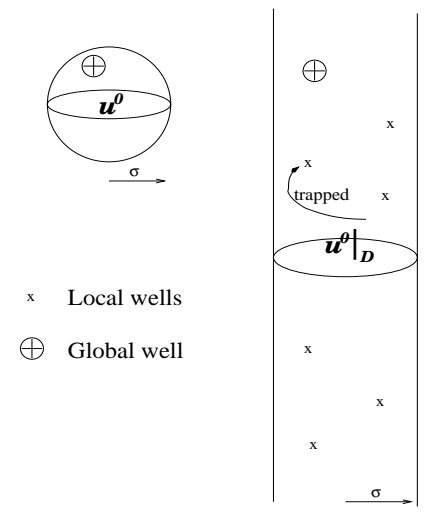

FIG. 4.1. Visualization of the difference between segmentation and inpainting (see the text explanation).
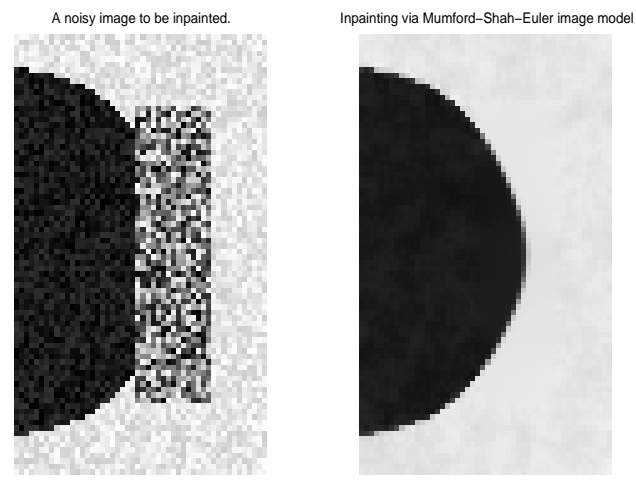

FIG. 4.2. With Euler's elastica curve model embedded inside, the inpainting model (4.3) based on the Mumford-Shah-Euler image model is able to restore smoother missing edges and yield more natural visual effect than the Mumford-Shah image model, as apparent from the comparison with Figure 3.1.

based on the Mumford-Shah image model [37], and its high-order correction, the MumfordShah-Euler image model. Our work extends and deepens the recent works of Tsai, Yezzi, and Willsky [43], and Chan and Shen [10].

We have explained from the viewpoint of image processing and vision analysis why the Mumford-Shah image model performs very well for segmentation and denoising, yet is insufficient for the inpainting application. Following Mumford's proposal of Euler's elastica curve model in computer vision [35], and its recent applications by Masnou and Morel [33], and Chan, Kang, and Shen [9], we propose to incorporate the elastica curve model into the Mumford-Shah image model to have a more effective image model for inpainting, for which we have coined the name - the Mumford-Shah-Euler image model.

We have introduced computational schemes for our inpainting models, based on the level-set method of Osher and Sethian [39], and the $\Gamma$-convergence approximation of Ambrosio and Tortorelli [1,2], and De Giorgi [18]. We have explained why working with an 

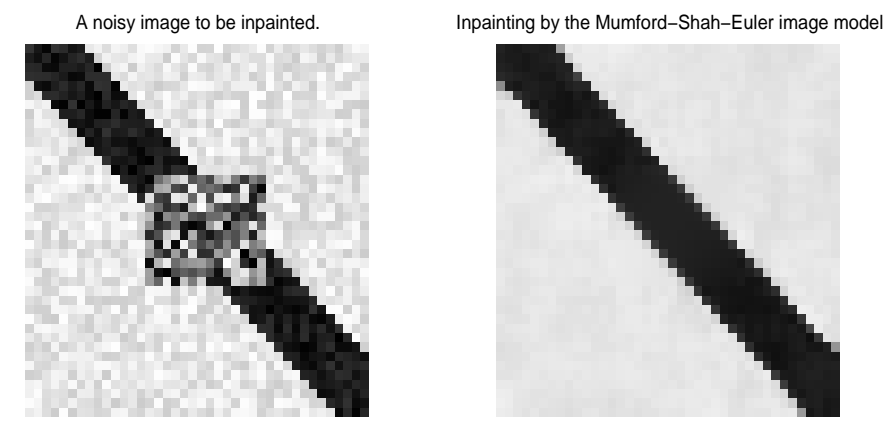

FIG. 4.3. By increasing the weight $\beta$ against the curvature term in the Mumford-Shah-Euler image model (4.2), the resulting inpainting model (4.3) is able to make a long-distance connection of separated parts. An inpainting model based on the Mumford-Shah image model only would have failed to make the connection, as already illustrated in Figure 3.2.

edge signature function $z$, instead of directly with the edge set $\Gamma$ itself, is more suitable for inpainting than for segmentation. Numerical schemes based on the $\Gamma$-convergence approximation are much simpler and converge faster. We thus have found a natural application for the high order $\Gamma$-convergence theory of De Giorgi, and its recent numerical implementation by March and Dozio [30, 31].

Our future research along this direction will be mainly targeted at solving the problem of energy wells of the inpainting energy, as discussed in the end of Section 4.

Acknowledgments. We owe enormous gratitude to Dr. Riccardo March for broadening our knowledge on computational $\Gamma$-convergence and numerous discussions; and to Dr. Marcelo Bertalmio and Prof. Guillermo Sapiro for generously teaching us the inpainting topic. We are happy to thank Institute of Mathematics and its Applications (IMA), and Prof. Willad Miller, Peter Olver, and Fadil Santosa for their support during this project. In addition, Selim Esedoglu benefited immensely from his recent visit to IAC in Rome, and would also like to thank his former advisor Robert V. Kohn for continuing support and encouragement. Jianhong (Jackie) Shen would like to thank all the following people for making the "vision" of the current paper possible: Prof. Gilbert Strang, Tony Chan, Stan Osher, Lumi Vese, SungHa Kang, Li-Tien Cheng, Dan Kerstan, David Mumford, Jean-Michel Morel, Stu Geman, Bob Gulliver, Rachid Deriche, and the Institute of Pure and Applied Mathematics (IPAM) at UCLA.

\section{REFERENCES}

[1] L. Ambrosio and V. M. Tortorelli. Approximation of functionals depending on jumps by elliptic functionals via $\Gamma$-convergence. Comm. Pure Appl. Math., 43:999-1036, 1990.

[2] L. Ambrosio and V. M. Tortorelli. On the approximation of free discontinuity problems. Boll. Un. Mat. Ital., 
6-B:105-123, 1992.

[3] S. Armstrong, A. Kokaram, and P.J.W. Rayner. Nonlinear interpolation of missing data using min-max functions. IEEE Int. Conf. Nonlinear Signal and Image Processings, 1997.

[4] C. Ballester, M. Bertalmio, V. Caselles, G. Sapiro, and J. Verdera. Filling-in by joint interpolation of vector fields and grey levels. IEEE Trans. Image Process., 10(8):1200-1211, 2001.

[5] M. Bertalmio, A. L. Bertozzi, and G. Sapiro. Navier-Stokes, fluid dynamics, and image and video inpainting. IMA Preprint 1772 at: www.ima.umn.edu/preprints/jun01, Juun, 2001.

[6] M. Bertalmio, G. Sapiro, V. Caselles, and C. Ballester. Image inpainting. Computer Graphics, SIGGRAPH 2000, July, 2000.

[7] G. Birkhoff and C. R. De Boor. Piecewise polynomial interpolation and approximation. In H. Garabedian, editor, Approximation of Functions, pages 164-190. Elsevier, 1965.

[8] V. Caselles, J.-M. Morel, and C. Sbert. An axiomatic approach to image interpolation. IEEE Trans. Image Processing, 7(3):376-386, 1998.

[9] T. F. Chan, S.-H. Kang, and J. Shen. Euler's elastica and curvature based inpaintings. UCLA CAM Report 2001-12 at: www.math.ucla.edu/imagers; submitted to SIAM J. Appl. Math., 2001.

[10] T. F. Chan and J. Shen. Mathematical models for local non-texture inpaintings. SIAM J. Appl. Math., in press, 2001.

[11] T. F. Chan and J. Shen. Morphologically invariant PDE inpaintings. UCLA CAM Report 2001-15 at: www.math.ucla.edu/imagers; submitted to IEEE Trans. Image Process., 2001.

[12] T. F. Chan and J. Shen. Non-texture inpainting by curvature driven diffusions (CDD). J. Visual Comm. Image Rep., to appear, 2001.

[13] T. F. Chan and J. Shen. Variational restoration of non-flat image features: models and algorithms. SIAM J. Appl. Math., 61(4):1338-1361, 2001.

[14] T. F. Chan and L. Vese. A level set algorithm for minimizing the Mumford-Shah functional in image processing. UCLA CAM Report 2000-13 at: www.math.ucla.edu/rimagers, 2000.

[15] L. Chanas, J. P. Cocquerez, and J. Blanc-Talon. Highly degraded sequences restoration and inpainting. Preprint, 2001.

[16] G. Emile-Male. The Restorer's Handbook of Easel Painting. Van Nostrand Reinhold, New York, 1976.

[17] S. Geman and D. Geman. Stochastic relaxation, Gibbs distributions, and the Bayesian restoration of images. IEEE Trans. Pattern Anal. Machine Intell., 6:721-741, 1984.

[18] E. De Giorgi. Some remarks on $\Gamma$-convergence and least squares methods. In G. Dal Maso and G. F. Dell'Antonio, editors, Composite Media and Homogenization Theory, pages 135-142. Birkhauser, Boston, MA, 1991.

[19] E. Giusti. Minimal Surfaces and Functions of Bounded Variation. Birkhäuser, Boston, 1984.

[20] G. H. Golub and J. M. Ortega. Scientific Computing and Differential Equations. Academic Press, 1992.

[21] H. Igehy and L. Pereira. Image replacement through texture synthesis. Proceedings of 1997 IEEE Int. Conf. Image Processing.

[22] K.-H. Jung, J.-H. Chang, and C. W. Lee. Error concealment technique using data for block-based image coding. SPIE, 2308:1466-1477, 1994.

[23] G. Kanizsa. Organization in Vision. Praeger, New York, 1979.

[24] D. C. Knill and W. Richards. Perception as Bayesian Inference. Cambridge Univ. Press, 1996.

[25] A. C. Kokaram, R. D. Morris, W. J. Fitzgerald, and P. J. W. Rayner. Detection of missing data in image sequences. IEEE Trans. Image Process., 11(4):1496-1508, 1995.

[26] A. C. Kokaram, R. D. Morris, W. J. Fitzgerald, and P. J. W. Rayner. Interpolation of missing data in image sequences. IEEE Trans. Image Process., 11(4):1509-1519, 1995.

[27] W. Kwok and H. Sun. Multidirectional interpolation for spatial error concealment. IEEE Trans. Consumer Electronics, 39(3), 1993.

[28] J. Langer and D. A. Singer. The total squared curvature of closed curves. J. Diff. Geom., 20:1-22, 1984. 
[29] A. E. H. Love. A Treatise on the Mathematical Theory of Elasticity. Dover, New York, 4th ed., 1927.

[30] R. March. Visual reconstruction with discontinuities using variational methods. Image Vision Comput., 10:30-38, 1992.

[31] R. March and M. Dozio. A variational method for the recovery of smooth boundaries. Image Vision Comput., 15:705-712, 1997.

[32] A. Marquina and S. Osher. Lecture Notes in Computer Science, volume 1682, chapter "A new time dependent model based on level set motion for nonlinear deblurring and noise removal", pages 429-434. 1999.

[33] S. Masnou and J.-M. Morel. Level-lines based disocclusion. Proceedings of 5th IEEE Int'l Conf. on Image Process., Chicago, 3:259-263, 1998.

[34] J. J. Moré and Z. Wu. Issues in large-scale global molecular optimization. In L. T. Biegler, T. F. Coleman, A. R. Conn, and F. N. Santosa, editors, Large-Scale Optimization with Applications, pages 99-121. Springer, New York, 1997.

[35] D. Mumford. Elastica and computer vision. In C. L. Bajaj, editor, Algebraic Geometry and its Applications, pages 491-506. Springer-Verlag, New York, 1994.

[36] D. Mumford. Geometry Driven Diffusion in Computer Vision, chapter "The Bayesian rationale for energy functionals", pages 141-153. Kluwer Academic, 1994.

[37] D. Mumford and J. Shah. Optimal approximations by piecewise smooth functions and associated variational problems. Comm. Pure Applied. Math., XLII:577-685, 1989.

[38] M. Nitzberg, D. Mumford, and T. Shiota. Filtering, Segmentation, and Depth. Lecture Notes in Comp. Sci., Vol. 662. Springer-Verlag, Berlin, 1993.

[39] S. Osher and J. A. Sethian. Fronts propagating with curvature-dependent speed: Algorithms based on Hamilton-Jacobi formulations. J. Comput. Phys., 79(12), 1988.

[40] L. Rudin and S. Osher. Total variation based image restoration with free local constraints. Proc. 1st IEEE ICIP, 1:31-35, 1994.

[41] L. Rudin, S. Osher, and E. Fatemi. Nonlinear total variation based noise removal algorithms. Physica D, 60:259-268, 1992.

[42] G. Strang. Introduction to Applied Mathematics. Wellesley-Cambridge Press, MA, 1993.

[43] A. Tsai, Jr. A. Yezzi, and A. S. Willsky. Curve evolution implementation of the Mumford-Shah functional for image segmentation, denoising, interpolation and magnification. IEEE Trans. Image Process., 10(8):1169-1186, 2001.

[44] S. Walden. The Ravished Image. St. Martin's Press, New York, 1985.

[45] L.-Y. Wei and M. Levoy. Fast texture synthesis using tree-structured vector quantization. Preprint, Computer Science, Stanford University, 2000; Also in Proceedings of SIGGRAPH 2000. 